\title{
New Labelling Technology for Molecular Probes Applied to the Ligation Detection Reaction-Universal Array System
}

\author{
Andrea Lauri $\cdot$ Stefania Chessa $\cdot$ Marta Raschetti • \\ Bianca Castiglioni • Paola Mariani • \\ Alexandre R. Caetano
}

Published online: 25 June 2010

(C) Springer Science+Business Media, LLC 2010

\begin{abstract}
The ligation detection reaction (LDR) associated with universal arrays (UA) uses a fluorescently labelled probe (DP) and a Zip Code-extended probe to detect single nucleotide polymorphisms in DNA target sequences. When used for genotyping, the LDR-UA technique uses two DPs, each specific to an allele and labelled with a different fluorophore. The fluorescent signals are processed to calculate the genotype. The uneven decay of fluorophores due to ageing and freezing/thawing cycles and the consequent unequal fluoresce level can lead to erroneous genotype calls. To circumvent this problem, an indirect labelling strategy was developed based on the substitution of the fluorophore with allele-specific $22 \mathrm{bp}$ universal labelling sequences (ULS). Labelling is achieved with fluorescently labelled oligos complementary to the ULS (cULS). The strategy improved the uniformity in probe labelling, and generated results comparable to those
\end{abstract}

\author{
A. Lauri $(\bowtie) \cdot$ P. Mariani \\ Parco Tecnologico Padano, Lodi, Italy \\ e-mail: andrea.lauri@ tecnoparco.org \\ P. Mariani \\ e-mail: paola.mariani@tecnoparco.org \\ S. Chessa - M. Raschetti - B. Castiglioni \\ IBBA-CNR, Milan, Italy \\ e-mail: chessa@ibba.cnr.it \\ M. Raschetti \\ e-mail: raschetti@ibba.cnr.it \\ B. Castiglioni \\ e-mail: casti@ibba.cnr.it
A. R. Caetano
Embrapa Recursos Genéticos e Biotecnologia, Brasília DF,
Brazil \\ e-mail: acaetano@cenargen.embrapa.br
}

using direct-labelled probes, as shown by genotyping 22 polymorphic sites in 70 samples with both strategies. This method can be easily implemented in the routine screening with LDR-UA or other techniques. Moreover, the approach results in a significant cost reduction over traditional direct labelling, and offers the possibility to interchange fluorophores and to increase the fluorescent signal by using multiple-labelled cULS.

Keywords Fluorescent labelling .

Ligation detection reaction - Genotyping - SNP .

Universal array

\section{Introduction}

Fluorescent dyes are frequently used to label molecules in lab procedures, and their use is gradually increasing across all molecular biology fields to replace other more dangerous alternatives, such as radioactive-labelled molecules. The ligation detection reaction coupled to universal array (LDR-UA) is a molecular technique that allows the detection of single nucleotide polymorphisms (SNPs) and takes advantage of fluorescently labelled probes [1].

The LDR-UA procedure uses two probes: a fluorescently labelled probe, also known as discriminating probe (DP), and the common probe (CP). The two probes are designed to anneal juxtaposed to the target site on singlestranded DNA, with DP annealing at the $5^{\prime}$ end of CP and DP designed with the last base corresponding to one of the SNP alleles. When there is perfect complementarity between the $3^{\prime}$ end of the DP and the target DNA, the two molecules can be ligated together.

The CP has at its 3' end a 25-bp "Zip Code" sequence, unique for each DP/CP probe set. Inverse complement of 


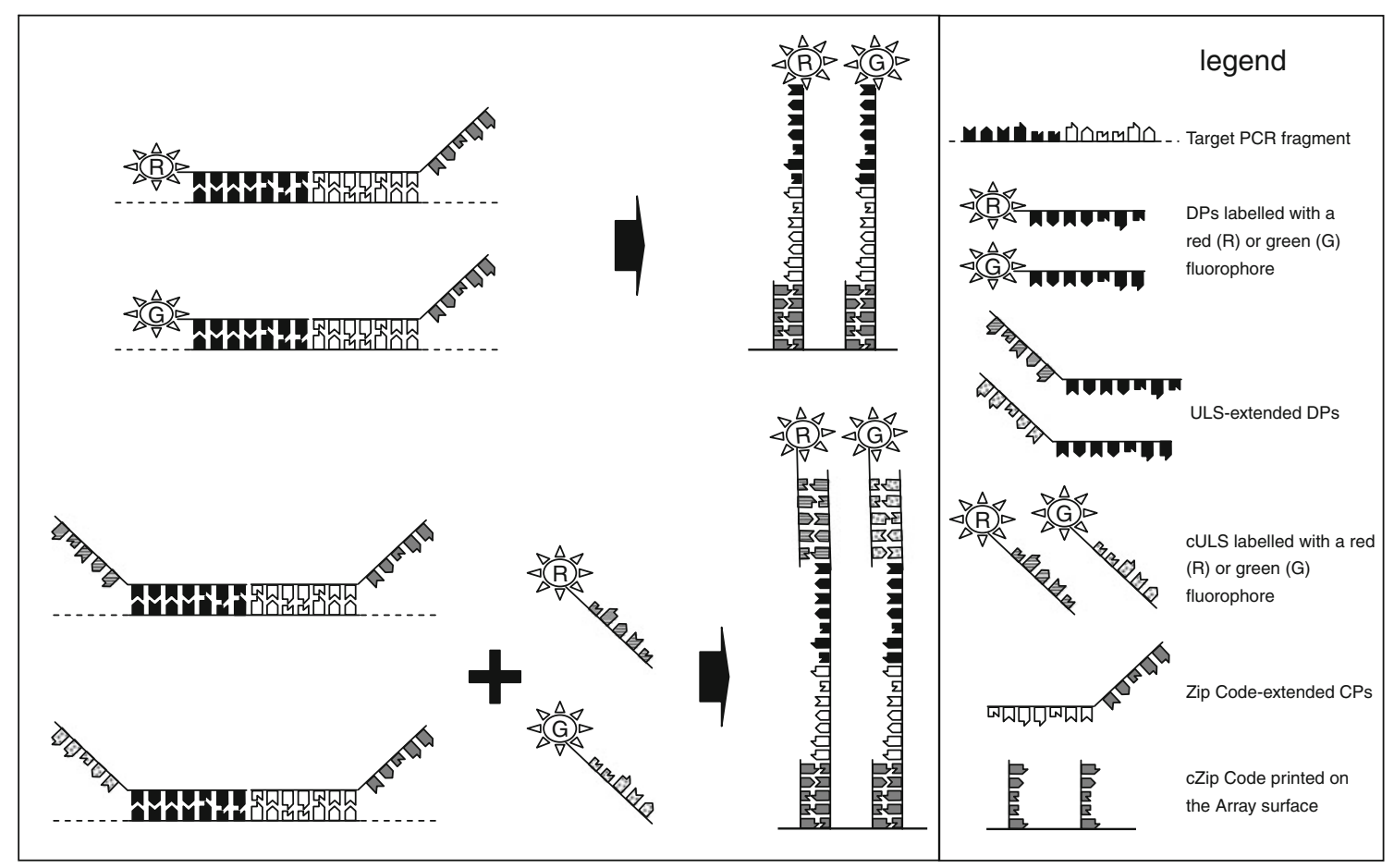

Fig. 1 The indirect labelling strategy is compared to the direct labelling approach. $U L S$ universal labelling sequence, $c U L S$ complementary of ULS, $D P$ discriminating probe, $C P$ common probe

the Zip Codes (cZIP Codes) are printed onto the UA to which the ligated CP-DP can be hybridised then detected and quantified by array scanning.

To discriminate between SNP alleles, DPs differing in the discriminating nucleotides are labelled with fluorophores of different colours. As SNPs are usually biallelic, two colours are typically used per SNP, and 40-50 SNPs can be interrogated simultaneously on the UA.

The LDR-UA has been shown to be a flexible and robust SNP genotyping method [2, 3]. However, the approach has a relatively high cost, with respect to other genotyping platforms. For instance, LDR-UA is cost-effective if compared to a TaqMan (Applied Biosystems, USA) approach investigating more than ten polymorphisms and with the Golden Gate technology (Illumina, USA) when assaying less than 45 . The major problem with the LDRUA approach is the natural decay of fluorophores which sensibly reduces the fluorescence emission even after a relatively short lag of time (a few months). Mixing different probe sets to genotype different polymorphic sites makes this technique highly flexible. However, mixing of probes with fluorophores of different ages will result in biased signal intensities and potentially genotyping errors.

The work presented describes the development of a strategy to uniformly label molecular probes which generates high-quality results and simultaneously lowers labelling costs. The approach uses an allele-specific, 22bp-labelled universal probe named universal labelling sequence (ULS). The DPs carry the complement of the ULS (cULS) and are then indirectly labelled by hybridisation with the ULS (Fig. 1).

The classical labelling method and the indirect labelling strategy were compared resulting in $100 \%$ concordance in genotype calls for the 22 investigated polymorphic sites in the 70 analysed samples. Expected positive impacts on the experimental costs and on the LDR-UA system performance are discussed.

\section{Materials and Methods}

All reagents used in the procedures reported in this section were purchased from Sigma-Aldrich (St. Louis, MO, USA), if not expressly indicated.

\section{Polymerase Chain Reaction (PCR)}

Gene polymorphisms investigated in this study and the respective PCR primers are the same as described by Chessa et al. [3]. DNA samples were extracted from 70 blood samples of the Italian Brown bovine breed. PCR was performed with $50 \mathrm{ng}$ genomic DNA in a final volume of $50 \mu \mathrm{l}$. Half of the PCR reaction was loaded onto a $1.2 \%$ EtBr-stained agarose gel to check for the presence of amplification products. 
The PCR products were purified by Sigma PCR purification kit, and DNA was eluted in $50 \mu \mathrm{l}$ of water.

\section{Molecular Probes and cULS/ULS Design}

Some constrains were adopted in the choice of the cULS/ ULS sequence. Short sequences (22 bp) were preferred to reduce the chance of secondary structure formation and to keep cost-effectiveness. The formation of secondary structure was calculated with GeneRunner (Hastings Software, USA), and the melting temperature was estimated by OligoCalc [4]. Sequences were compared with the whole NCBI dataset by BLAST (http://blast.ncbi.nlm.nih.gov/ Blast.cgi) to exclude the possibility of unwanted annealing.

The cULS sequences tested were Cy3-AGTGGGCG AACGACAGGGAGGCG and Cy5-ACGCTCGGCTCGC ATCGCATCGG, and the respective ULS, fused to DPs, were CGCCTCCCTGTCGTTCGCCCAC and CCGATG CGATGCGAGCCGAGCG, as reported in Table 1. CP sequences were identical to those used by Chessa et al. [3].

All probes and cULS were purchased from VWR (Milan, Italy).

\section{Ligation Detection Reaction and Universal Array}

Hybridisation

The LDR was carried out in a volume of $20 \mu \mathrm{l}$. The reaction mix included: 1 unit of Pfu Ligase (Stratagene), $2 \mu \mathrm{l}$ of $10 \times$ buffer, 50-400 ng of purified PCR product and $\mathrm{CP}$ and DP probes to a final concentration of $50 \mathrm{nM}$ each. The mix was first denatured for $10^{\prime}$ at $94^{\circ} \mathrm{C}$, followed by $30^{\prime \prime}$ at $94^{\circ} \mathrm{C}$ and $4^{\prime}$ at $65^{\circ} \mathrm{C}$, repeated for 30 cycles.

The LDR products were then added to $45 \mu$ l hybridisation buffer $(\mathrm{NaCl} 1.10 \mathrm{M}, 0.11 \mathrm{M}$ sodium citrate, $7 \mu \mathrm{g}$ Salmon Sperm DNA) and to cULS. The final concentration of cULS in the hybridisation mix was empirically set at 500 $\mathrm{nM}$ each; higher concentrations would not yield a stronger signal (Fig. 2b). The solution was denatured at $94^{\circ} \mathrm{C}$ for $3^{\prime}$ and then chilled on ice.

Samples were then hybridised on UA at $65^{\circ} \mathrm{C}$ for $2 \mathrm{~h}$. The UA was then washed in SSC $2 \times(0.3 \mathrm{M} \mathrm{NaCl}, 0.03 \mathrm{M}$ sodium citrate) and $0.1 \%$ SDS (European Patent 08161325.9 pending).

\section{Universal Array Preparation}

Phenylene-diisothiocyanate (PDITC)-activated chitosan glass slides were used as surfaces [5]. Universal array were prepared as described in [2], using a MicroGrid II Compact (Biorobotics, UK) contact pin spotter, equipped with silicon microarray pins (Parallel Synthesis Technologies, Santa Clara, CA, USA).
UA Scanning and Fluorescent Signal Acquisition

Hybridised UAs were scanned with a ScanArray Lite laser scanning system and ScanArray Express Microarray Analysis System 2.1 software (PerkinElmer Life Sciences, Boston, MA, USA). The acquired image was then analysed to quantify the fluorescence intensity according to procedures described by Chessa et al. [3].

Signals from different channels were balanced according to values from reference probes (CSN2-UNI-Cy3 and CSN2-UNI-Cy5) to even fluorophore disparities. After adjustments, genotypes for each locus/spot were determined by dividing intensity values from one of the two channels ( $\mathrm{Cy} 3$, in the present work) by the total intensity of the spot (i.e. the sum of signals from the two channels). Values included between 0.35 and 0.75 represent a heterozygote, whereas values below 0.35 or above 0.75 indicate homozygotes.

\section{Results}

In a recent publication, Chessa et al. [3] used a LDR-UA approach to detect mutations in genes having a known effect on milk quality. With the aim of improving probe signal to background noise ratio and significantly reducing costs, we used an indirect probe-labelling strategy, based on the annealing of a labelled oligo (cULS) to the $5^{\prime}$ end extension of the DP (ULS), to assay the same loci.

Two cULS/ULS probe pairs were designed to reach the highest performance in terms of oligonucleotide annealing with the shortest sequence length. Both pairs have a melting temperature of $65^{\circ} \mathrm{C}$, corresponding to the hybridisation temperature of the LDR products on the UA. In addition, cULS were also optimised to be free of predicted secondary structures, such as hairpin loops or dimers at temperatures above $30^{\circ} \mathrm{C}$. No nucleotide sequences at the NCBI database showed more than $82 \%$ sequence identity with the cULS sequences used, suggesting that the unspecific hybridisations of cULS with any DNA molecules other than ULS is unlikely at $65^{\circ} \mathrm{C}$. In addition, probe design included an adenine (A) spacer between the fluorophore and the last base of the cULS to reduce the quenching effect of guanidine $(\mathrm{G})$.

Experiments were performed with cULS labelled either at the $5^{\prime}$ or at the $3^{\prime}$ end to test the efficiency of the indirect labelling method. Normalisation reference probes CSN2UNI-Cy3 and CSN2-UNI-Cy5 were labelled with $500 \mathrm{mM}$ cULS carrying the Cy3 or Cy5 fluorophore either at the $3^{\prime}$ or at the $5^{\prime}$ end. The best performance was registered with the $5^{\prime}$-labelled cULS that resulted in labelling efficiencies 
Table 1 List of the discriminating probes used in the present work and modified from Chessa et al. [3]

\begin{tabular}{|c|c|}
\hline Probe name & Discriminating probe sequence \\
\hline CSN1S1-17807-A & CGCCTCCCTGTCGTTCGCCCAC_TCTGACATCCCTAATCCCATTGGCTCTGAGAACAGTGA \\
\hline CSN1S1-17807-G & CCGATGCGATGCGAGCCGAGCG_TCTGACATCCCTAATCCCATTGGCTCTGAGAACAGTGG \\
\hline CSN2-6690-G & CGCCTCCCTGTCGTTCGCCCAC_TTTTAACCAGAAAATTGAGAAGTTTCAGAGTGAGG \\
\hline CSN2-6690-A & CCGATGCGATGCGAGCCGAGCG_TTTTAACCAGAAAATTGAGAAGTTTCAGAGTGAGA \\
\hline CSN2-8101-C & CGCCTCCCTGTCGTTCGCCCAC_AGTCTCTAGTCTATCCCTTCCCTGGICCCATCCC \\
\hline CSN2-8101-A & CCGATGCGATGCGAGCCGAGCG_AGTCTCTAGTCTATCCCTTCCCTGGICCCATCCA \\
\hline CSN2-8178-A & CGCCTCCCTGTCGTTCGCCCAC_GTGGTGGTGCCGCCTTTCCTTCAGCCTGAAGTAA \\
\hline CSN2-8178-C & CCGATGCGATGCGAGCCGAGCG_GTGGTGGTGCCGCCTTTCCTTCAGCCTGAAGTAC \\
\hline CSN2-8219-C & CGCCTCCCTGTCGTTCGCCCAC_GTCTCCAAAGTGAAGGAGGCTATGGCTCCTAAGCAC \\
\hline CSN2-8219-A & CCGATGCGATGCGAGCCGAGCG_GTCTCCAAAGTGAAGGAGGCTATGGCTCCTAAGCAA \\
\hline CSN2-8267-C & CGCCTCCCTGTCGTTCGCCCAC_CCTTCCCTAAATATCCAGTTGAGCCCTTTACTGAAAGC \\
\hline CSN2-8267-G & CCGATGCGATGCGAGCCGAGCG_CCTTCCCTAAATATCCAGTTGAGCCCTTTACTGAAAGG \\
\hline CSN3-12951-G & CGCCTCCCTGTCGTTCGCCCAC_CCCAGCCAACTACCATGGGCACG \\
\hline CSN3-12951-A & CCGATGCGATGCGAGCCGAGCG_GCCCAGCCAACTACCATGGCACA \\
\hline CSN3-13065-C & CGCCTCCCTGTCGTTCGCCCAC_TAGTGGTCAGCCTACAAGTACACCTAC \\
\hline CSN3-13065-T & CCGATGCGATGCGAGCCGAGCG_CTAGTGGTGAGCCTACAAGTACACCTAT \\
\hline CSN3-13068-C & CGCCTCCCTGTCGTTCGCCCAC_GTGAGCCTACAAGTACACCTACCAC \\
\hline CSN3-13068-T & CCGATGCGATGCGAGCCGAGCG_TGGTGAGCCTACAAGTACACCTACCAT \\
\hline CSN3-13104-A & CGCCTCCCTGTCGTTCGCCCAC_GTAGAGAGCACTGTAGCTACTCTAGAAGA \\
\hline CSN3-13104-C & CCGATGCGATGCGAGCCGAGCG_GAGAGCACTGTAGCTACTCTAGAAGC \\
\hline CSN3-13124-A & CGCCTCCCTGTCGTTCGCCCAC_GCTCTAGAAGATTCTCCAGAAGTTATTGAGA \\
\hline CSN3-13124-G & CCGATGCGATGCGAGCCGAGCG_CTCTAGAAGATTCTCCAGAAGTTATTGAGG \\
\hline CSN3-13165-A & CGCCTCCCTGTCGTTCGCCCAC_ATCAACACAGTCCAAGTTACTTCAACTGCA \\
\hline CSN3-13165-G & CCGATGCGATGCGAGCCGAGCG_CAACACAGTCCAAGTTACTTCAACTGCG \\
\hline LGB-3982-TGA & CGCCTCCCTGTCGTTCGCCCAC_CGTGGCTCAGAAAGCAGCTGTCTTTCAGGGAGAATGA \\
\hline LGB-3982-CGG & CCGATGCGATGCGAGCCGAGCG_TGGCTCAGAAAGCAGCTGTCTTTCAGGGAGAACGG \\
\hline LGB-5174-C & CGCCTCCCTGTCGTTCGCCCAC_TCACTTTCCTCCCGTCTTGATCTCTTCCAGCCTTGAAC \\
\hline LGB-5174-T & CCGATGCGATGCGAGCCGAGCG_TCACTTTCCTCCCGTCTTGATCTCTTCCAGCCTTGAAT \\
\hline LGB-5223-T & CGCCTCCCTGTCGTTCGCCCAC_CCTTGTGCTGGACACCGACTACAAAAAGTACCTGCTCT \\
\hline LGB-5223-G & CCGATGCGATGCGAGCCGAGCG_CTTGTGCTGGACACCGACTACAAAAAGTACCTGCTCG \\
\hline LGB-5261-GGT & CGCCTCCCTGTCGTTCGCCCAC_AGAACAGTGCTGAGCCCGAGCAAAGCCTGGT \\
\hline LGB-5261-KGC & CCGATGCGATGCGAGCCGAGCG_AGAACAGTGCTGAGCCCGAGCAAAGCCTKGC \\
\hline Bov-A2-8371-A & CGCCTCCCTGTCGTTCGCCCAC_TAGACTGCAGTCCATGGGGTCACTAA \\
\hline Bov-A2-8371-G & CCGATGCGATGCGAGCCGAGCG_GACTGCAGTCCATGGGGTCACTAG \\
\hline PromCSN1S1-289-A & CGCCTCCCTGTCGTTCGCCCAC_TTGAAGACCCCATTTTGTCCCAAGAATTTCA \\
\hline PromCSN1S1-289-C & CCGATGCGATGCGAGCCGAGCG_GAAGACCCCATTTTGTCCCAAGAATTTCC \\
\hline PromCSN1S1-395-396 & CGCCTCCCTGTCGTTCGCCCAC_CCATAAATCTAGGGTTTTGTTGGGGTTTTTT \\
\hline PromCSN1S1-Del 395-396 & CCGATGCGATGCGAGCCGAGCG_CATAAATCTAGGGTTTTGTTGGGGTTTTTTG \\
\hline PromCSN1S1-Del 397-400 & CCGATGCGATGCGAGCCGAGCG_CTAGGGTTTTGTTGGGGTTTTTTTTGTTTG \\
\hline PromCSN1S1-397-400 & CGCCTCCCTGTCGTTCGCCCAC_ATAAATCTAGGGTTTTGTTGGGGTTTTTTTTGTT \\
\hline PromCSN1S1-444-A & CGCCTCCCTGTCGTTCGCCCAC_CAATGCCATTCCATTTCCTGTATAATGAGTCA \\
\hline PromCSN1S1-444-G & CCGATGCGATGCGAGCCGAGCG_ATGCCATTCCATTTCCTGTATAATGAGTCG \\
\hline PromCSN1S1-484-G & CGCCTCCCTGTCGTTCGCCCAC_GTAAACTCTCCTTAGAATTTCTTGGGAGAG \\
\hline PromCSN1S1-484-C & CCGATGCGATGCGAGCCGAGCG_GTAAACTCTCCTTAGAATTTCTTGGGAGAC \\
\hline
\end{tabular}


Table 1 continued

\begin{tabular}{|c|c|}
\hline Probe name & Discriminating probe sequence \\
\hline CSN2-UNI-Cy3 & CGCCTCCCTGTCGTTCGCCCAC_GAAAGCAGTGCCCTATCCCCAGAGAGATATGCCCA \\
\hline CSN2-UNI-Cy5 & CCGATGCGATGCGAGCCGAGCG_GAAAGCAGTGCCCTATCCCCAGAGAGATATGCCCA \\
\hline cULS & Sequence \\
\hline cULS-Cy3 & AGTGGGCGAACGACAGGGAGGCG \\
\hline cULS-Cy5 & ACGCTCGGCTCGCATCGCATCGG \\
\hline
\end{tabular}

The probes were extended at their $5^{\prime}$ end with universal labelling sequences (ULS), reported before the underscore sign. The two complementary ULS sequences, labelled with a Cy3 or Cy5 fluorophores are also indicated

three times higher than cULS labelled at the $3^{\prime}$ end (Fig. 2a). The cULS thereafter used in the indirect labelling tests were marked at the $5^{\prime}$ end.

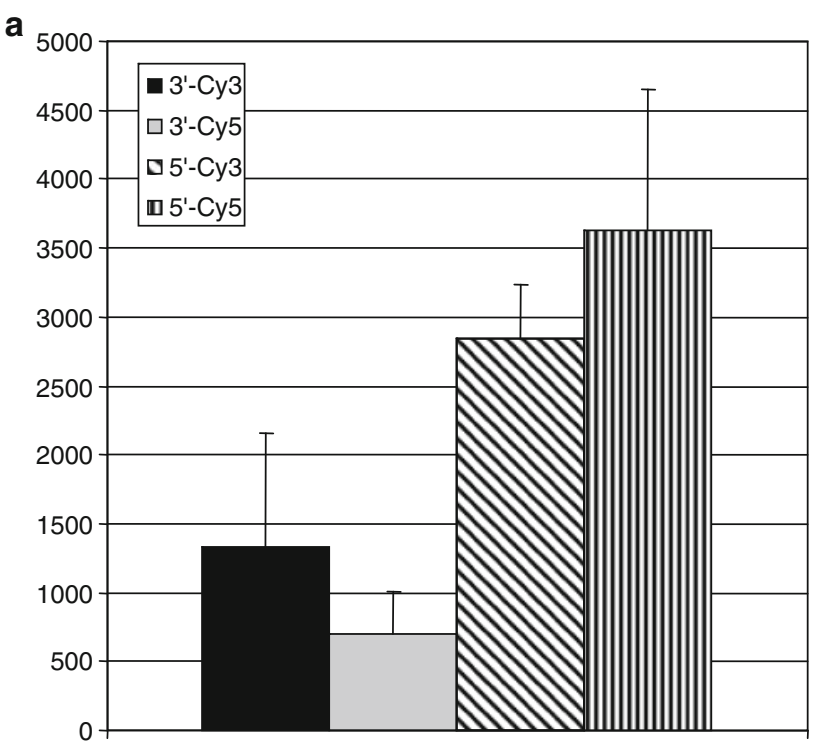

b

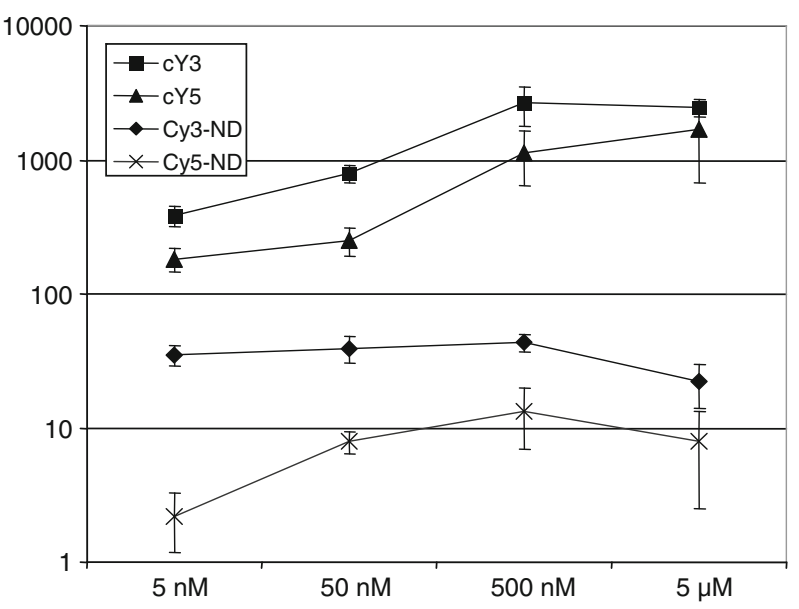

Fig. 2 a Comparison of probe-labelling efficiency with cULS labelled at the $5^{\prime}$ or $3^{\prime}$ end, with both Cy3 and Cy5 fluorophores. b Titration curve with increasing cULS concentration in presence $(\mathrm{Cy} 3$ and Cy5) or absence (Cy3-ND and Cy5-ND) of target DNA. Values are reported on a logarithmic scale
The optimal cULS concentration was empirically determined by testing various cULS dilutions. Experiments with concentrations of 5, 50, 500 and 5,000 nM of each cULS were performed. The fluorescence signal of the array spot corresponding to the CSN2-UNI-Cy3 and CSN2-UNI-Cy5 probes were used to construct titration curves for each fluorophore (Fig. 2b). The optimal cULS concentration resulted to be $500 \mathrm{nM}$, which corresponds to ten times the concentration of each DP. Higher cULS concentrations $(5 \mu \mathrm{M})$ did not bring a significant increment in spot fluorescence. Experiments performed with no target DNA were used to evaluate background fluorescence and showed low fluorescence signals. Background signals were well distinct from those registered in presence of target DNA, and did not show an increment with increasing cULS concentrations.

A comparative example of an experiment carried out with the direct and indirect labelling methods is shown in Fig. 3. The table below reports absolute fluorescence values for both experiments ( $\mathrm{Cy} 3$ and $\mathrm{Cy} 5$ columns) that were then processed as described [3], and the resulting allelic fraction is reported in the AFC35 column. In this example, the benchmark probe set (CSN2-UNI) used to balance the Cy3 and Cy5 channels had comparable fluorescent values for the Cy3 fluorophore either using the direct labelling strategy or the cULS/ULS method. Values for the Cy5 channel were higher for cULS/ULS than for the direct labelling. For the rest of the cULS/ULS probes, absolute values were always lower for $\mathrm{Cy} 3$ and for about half of the Cy5. Nevertheless, the heterozygote/homozygote state based on the allelic fraction values calculated for cULS/ULS was consistent with that reported for the direct labelling strategy.

The study was conducted on samples from 70 Italian Brown cows. Allelic fractions relative to the 22 interrogated polymorphic sites were calculated for the direct and indirect labelling methods (Fig. 4). Significant differences in allelic fraction values obtained with each method were observed in some loci (e.g. Bov-A2-8371, CSN2-8267 or CSN3-13068 heterozygote values). However, this variability in no case altered the correct genotyping of the sample as all values were contained in the defined heterozygote or homozygote range. 
Fig. 3 Comparative example of an experiment performed with cULS/ULS (left) and an experiment performed with directly labelled probes (right). The table reports absolute fluorescence values registered in the two experiments $(\mathrm{Cy} 3$ and Cy5 columns) and the relative calculated allelic fraction (AF C35). (Color figure online)

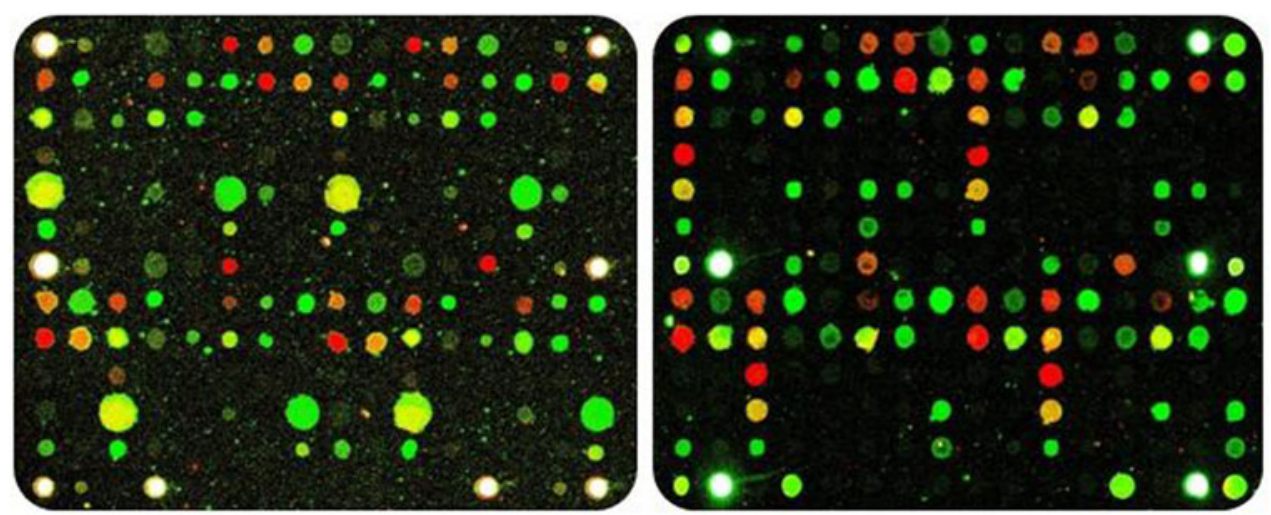

\begin{tabular}{l|rrr|rrr|}
\cline { 2 - 7 } $\begin{array}{l}\text { Polymorphism } \\
\text { name }\end{array}$ & \multicolumn{2}{c|}{ cULSIULS indirect labeling } & \multicolumn{2}{c|}{ Probe direct labeling } \\
& \multicolumn{1}{c}{ Cy3 } & Cy5 & AF C35 & Cy3 & Cy5 & AF C35 \\
CSN3-12951 & 276.25 & 94.75 & 0.80 & 2053.75 & 53.75 & 0.96 \\
CSN3-13065 & 284.375 & 68.5 & 0.85 & 425 & 56 & 0.83 \\
CSN3-13068 & 164.15 & 3579.5 & 0.06 & 634.5 & 2225.75 & 0.15 \\
CSN3-13104 & 267.6 & 2144 & 0.15 & 393.25 & 2702.75 & 0.08 \\
CSN3-13124 & 806.75 & 85.5 & 0.93 & 943.5 & 58.75 & 0.91 \\
CSN3-13165 & 103.25 & 3260.25 & 0.04 & 354.625 & 6433.65 & 0.03 \\
PromCSN1S1-289 & 5842.75 & 80.75 & 0.99 & 10282.5 & 60.25 & 0.99 \\
PromCSN1S1-Del-396 & 159.575 & 77.2 & - & 244.4 & 83.2 & - \\
PromCSN1S1-Del-397 & 96.5 & 508 & 0.21 & 162.75 & 1253.2 & 0.08 \\
PromCSN1S1-444 & 895.75 & 115.5 & 0.92 & 2646.75 & 89.5 & 0.95 \\
PromCSN1S1-484 & 12583.5 & 304.85 & 0.98 & 13734.25 & 130.2 & 0.99 \\
Bov-A2-8371 & 298 & 4726 & 0.08 & 1201 & 10816.75 & 0.07 \\
LGB-3982 & 2491.775 & 3283.25 & 0.52 & 10335 & 3884.25 & 0.63 \\
LGB-5174 & 10790.85 & 7007 & 0.68 & 11974 & 12717.75 & 0.37 \\
LGB-5223 & 561.25 & 108.75 & 0.88 & 1173.25 & 51.5 & 0.93 \\
LGB-5261 & 2956.5 & 1732.125 & 0.71 & 13711.5 & 10519.25 & 0.45 \\
CSN1S1-17807 & 1624 & 58.75 & 0.97 & 4236.75 & 79.5 & 0.97 \\
CSN2-6690 & 437 & 103.5 & 0.86 & 17125.75 & 145 & 0.99 \\
CSN2-8101 & 1659 & 225.45 & 0.91 & 6163.75 & 347.55 & 0.92 \\
CSN2-8178 & 839.3 & 100.8 & 0.92 & 2819.6 & 106.05 & 0.94 \\
CSN2-8219 & 270.2 & 84 & 0.81 & 282.75 & 38.75 & 0.82 \\
CSN2-8267 & 3281 & 140 & 0.97 & 5621 & 134 & 0.96 \\
CSN2-UNI & 3470.749 & 4886.55 & 0.50 & 3169.8 & 1991.85 & 0.50 \\
\hline
\end{tabular}

\section{Discussion}

A new method to indirectly label LDR-UA probes was developed with the aim of improving performance and reducing costs for medium-density genotyping (10-45 polymorphic sites). Indirect labelling was achieved with fluorescently marked $22 \mathrm{bp}$ oligonucleotides (cULS) that anneal to the corresponding $5^{\prime}$ end tail (ULS) on the discriminating probe (DP). The cULS/ULS sequence was designed not to form secondary structures that might interfere with specific annealing, and to hybridise to each other at standard LDR hybridisation temperatures $\left(65^{\circ} \mathrm{C}\right)$.
Unspecific annealing of the cULS with oligonucleotides spotted on the array or other DNA molecules used in these experiments was not registered, as the background signal registered in absence of target DNA was negligible, even at the highest cULS concentration $(5 \mu \mathrm{M}$; Fig. $2 \mathrm{~b})$. Future experiments are expected to be equally free of cross-hybridisations as cULS do not have high homology with any DNA sequence reported in the NCBI database.

Higher signal intensity was observed with the cULS labelled at the $5^{\prime}$ end compared with the $3^{\prime}$ end (Fig. 2a). This might be explained by a higher efficiency for $5^{\prime}$ end labelling protocol with respect to the protocol used to label 


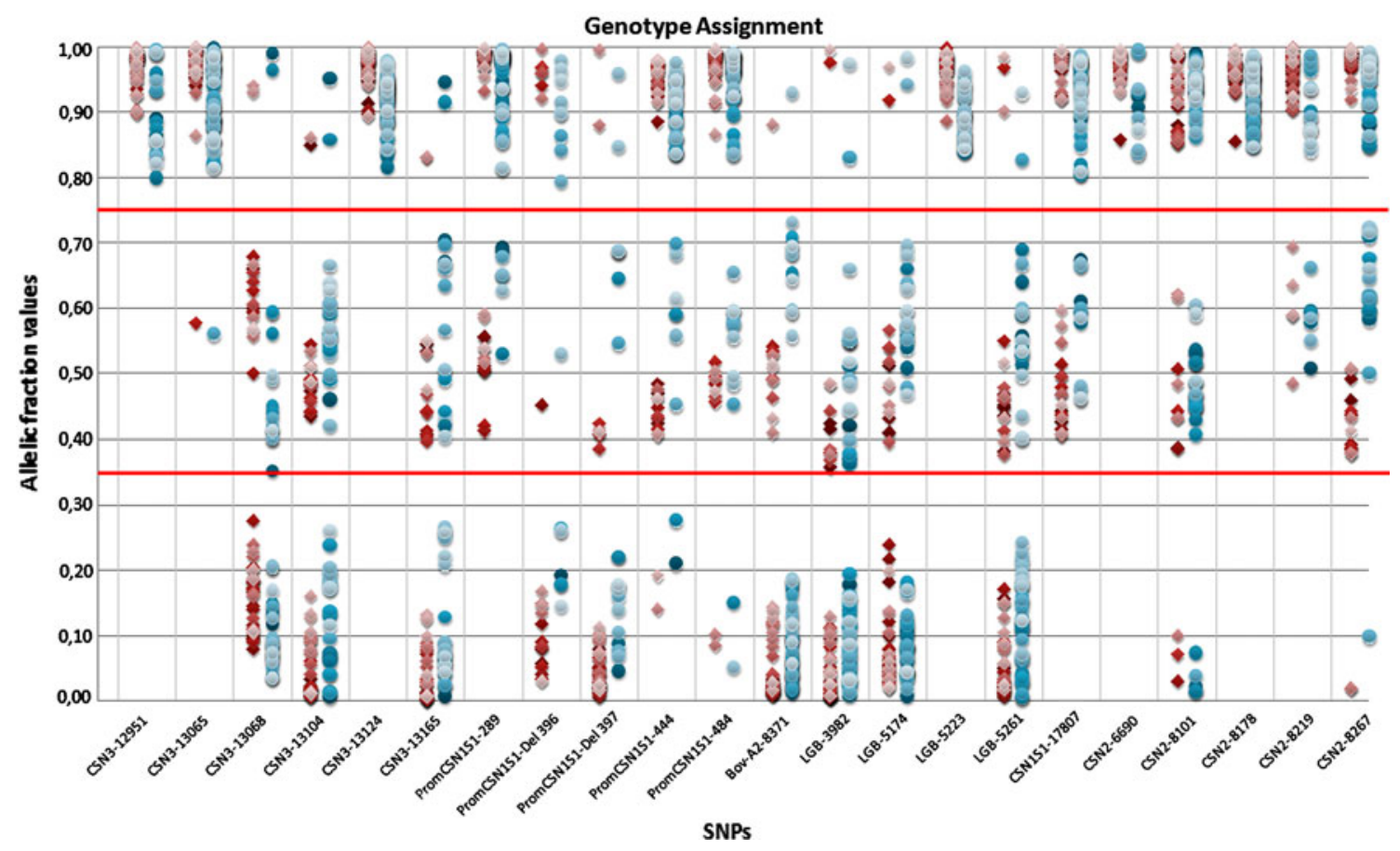

Fig. 4 Allelic fractions relative to 70 experiments are reported for each investigated polymorphic site. Squares and circles represent direct and indirect labelling, respectively

the $3^{\prime}$ end, which were different [6] (Thermo Fisher Scientific, personal communications).

Different dilutions of the cULS were tested with optimal results obtained at $500 \mathrm{nM}$ each. The titration curve suggests that at this concentration all cULS-binding sites (ULS) are saturated, as higher cULS concentrations would not increase the fluorescent signal (Fig. 2b).

Finally, extending the hybridisation time from $1 \mathrm{~h}$ [1] to $2 \mathrm{~h}$, resulted in a slight increase in the fluorescent signal (not shown). This modification of the original LDR-UA protocol did not bring an increment in background signals.

Genotyping using the cULS-labelled probes in LDR-UA was validated against the traditional direct-labelled approach. While a reduction of fluorescence intensity was observed in about $50 \%$ of the indirect labelling experiments, the final assessment of sample genotypes was not affected in any way (Fig. 3).

Experiments were conducted on a relevant number of samples (70), and the genotypes registered with the direct labelling method were always consistent with the cULS/ULS labelling method. In some of the polymorphisms tested (e.g. Bov-A2-8371, CSN2-8267 or CSN3-13068 heterozygote values), the data obtained with the two methods, although always included in the defined ranges, appeared to form two separate groups with a higher or lower trend. These differences could be due to the different labelling methodologies that, in the cited cases, introduced non-random bias in the calculation of the allelic fraction values. These biases could be corrected in the next genotyping experiments with the introduction of a correction coefficient specifically calculated for these probe sets. In other cases (e.g. PromCSN1S1Del397 heterozygote values), the observed bias could not be discussed due to the limited number of data.

Advantages are expected from the routine use of the cULS/ULS system, both in terms of improved performances and cost reduction. Fluorophore decay, i.e. light emission efficiency loss, is a phenomenon that results from ageing or repeated thawing/freezing cycles and is often experienced with LDR probes and other laboratory consumables [7]. The use of fluorophores of different ages or with unequal loss of efficiency can have important consequences in LDR-UA genotyping experiments. In fact, the loss of signal in one channel would unbalance the signal ratio and therefore lead to misinterpretations of the results. This is especially true when the investigated polymorphic site is heterozygote, and the expected yellow signal, resulting from the overlapping of the red and green channels, might shift towards one of the two colours. With the use of cULS/ULS, all probes are equally labelled, and the channel signal is a function only of the concentration of the DP, as the signal does not depend on the age or on the state of decay of single probes.

Although the cULS indirect labelling was tested on LDR probes, it can be implemented in many other technologies that require DNA labelling. For instance, microarray 
expression profile studies use labelled oligodT primers or marked deoxynucleotides for the universal reverse-transcription and simultaneous labelling of mRNA samples. The use of ULS-extended oligodT would make possible the indirect labelling of the reverse-transcribed sample.

The cULS that in turn reach a state of decay no longer compatible with experimental quality can be replaced with freshly labelled cULS at the sole cost of the new oligos and not of the whole DP set. Synthesis of cULSs with identical nucleotide sequences but with different dyes is also possible and creates assay flexibility without re-synthesis of the entire LDR probe set. This might be convenient in dye swapping control experiments, i.e. the repetition of a genotyping experiment with inverted fluorophores to rule out dye-specific effects [8]. Moreover, cULSs labelled with new fluorophores can be easily tested or the signal can be increased with the use of cULS carrying more fluorescent molecules, without changing all the DPs.

Comparing the price of directly labelled DP to the ULS extended DP, the latter allows savings of about $25 \%$ on the costs of the DP set. In fact, according to the pricelist of our supplier (VWR 2008, Milan), the cost of the ULS extension is about half of the cost of the fluorophore, i.e. about half of the price of the modified DP.

The cost of the two labelled cULS was minimal with respect to the cost of the whole DP set and corresponded to about $1 / 10$. The amount of cULS supplied is theoretically enough for 3,500 experiments.

In order to further reduce the procedure costs, unphosphorylated CPs were implemented. A kinase enzyme (NEB, Ipswich, USA) was used to add a phosphate to the $5^{\prime}$ end of CPs before the LDR. This "in house" phosphorylation resulted in no reduction of the reaction efficiency in experiment controls and contributed to reduce the cost of the CP set by $1 / 3$ (not shown).

Recently, Girigoswami et al. [9] published a different indirect labelling strategy for LDR-UA based on the tight biotin/streptavidin interaction. The method used modified CPs biotinylated at the $3^{\prime}$ and that were indirectly labelled with streptavidin linked to a fluorophore. Although the use of the biotin/streptavidin allowed the implementation of one fluorophore per experiment, authors showed that the system can be used to assess the allelic state of the investigated DNA by assigning a different Zip Code to each DP. The ratio of signals from the corresponding UA spots indicates the presence or absence of each allele in a similar fashion to the fluorophore intensity ratio described for the cULS/ULS system. However, the signal ratio from different UA spots can be biased by some factors such as the Zip Code sequence, which is different in each probe and can influence the annealing to the UA spot. Moreover, spot sizes can vary significantly in spotted microarrays, especially if printed with a pin spotter technology, even within the same microarray. The bias introduced with these factors can be hardly normalised and might lead to an erroneous output.

Conversely, the implementation of one Zip Code for the two DP labelled with different dyes, as described for the cULS/ULS, allows a better performance. In fact, differences imputable only to fluorophores can be easily balanced by introducing artificial heterozygous samples to be used as a benchmark.

Finally, the modification of oligonucleotides with biotin is only slightly cheaper than direct labelling, resulting in no real economic advantage.

The indirect labelling strategy presented here couples a reduced experiment budget with high performances. The method was tested with LDR-UA probes, but could be implemented with any kind of labelling, from expression microarrays to in situ detection, etc. Improvements are also possible and might concern modification of the cULS, for instance with locked nucleic acid (LNA) that stabilises the cULS/ULS duplex or with more and brighter fluorophores, as well as radioactive isotopes, etc.

\section{References}

1. Gerry, N. P., Witowski, N. E., Day, J., Hammer, R. P., Barany, G., \& Barany, F. (1999). Universal DNA microarray method for multiplex detection of low abundance point mutations. Journal of Molecular Biology, 292, 251-262.

2. Castiglioni, B., Rizzi, E., Frosini, A., Sivonen, K., Rajaniemi, P., Rantala, A., et al. (2004). Development of a universal microarray based on the ligation detection reaction and $16 \mathrm{~S}$ rrna gene polymorphism to target diversity of cyanobacteria. Applied and Environmental Microbiology, 70, 7161-7172.

3. Chessa, S., Chiatti, F., Ceriotti, G., Caroli, A., Consolandi, C., Pagnacco, G., et al. (2007). Development of a single nucleotide polymorphism genotyping microarray platform for the identification of bovine milk protein genetic polymorphisms. Journal of Dairy Science, 90, 451-464.

4. Kibbe, W. A. (2007). OligoCalc: an online oligonucleotide properties calculator. Nucleic Acids Research, 35, W43-W46.

5. Consolandi, C., Severgnini, M., Castiglioni, B., Bordoni, R., Frosoni, A., Battaglia, C., et al. (2006). A structured chitosanbased platform for biomolecule attachment to solid surfaces: application to DNA microarray preparation. Bioconjug Chem, 17, 371-377.

6. Hilario, E. (2004). End labeling procedures: an overview. Molecular Biotechnology, 28, 77-80.

7. Nagel, M., Richter, F., Haring-Bolivar, P., \& Kurz, H. (2003). A functionalized THz sensor for marker-free DNA analysis. Physics in Medicine and Biology, 48, 3625-3636.

8. Sanchez-Cabo, F., Prokesch, A., Thallinger, G. G., Pieler, R., Trajanoskie, Z., Butcher, P. D., Hinds, J., Holmes, L. E. A., Campbell, S. G., Ashe, M. P., Hubbard, S., Cho, K. H., Wolkenhauer, O. (2004) Assessing the efficiency of dye-swap normalization to remove systematic bias from two-color microarray data. Appl Bioinformatics, in press.

9. Girigoswami, A., Jung, C., Mun, H. Y., \& Park, H. G. (2008). PCR-free mutation detection of BRCA1 on a zip-code microarray using ligase chain reaction. Journal of Biochemical and Biophysical Methods, 70, 897-902. 